\title{
Snail-dependent repression of the RhoGEF pebble is required for gastrulation consistency in Drosophila melanogaster
}

\author{
Michael J. Murray • Tony D. Southall • Wenjie Liu • \\ Hamilton Fraval • Nirmal Lorensuhewa • \\ Andrea H. Brand • Robert Saint
}

Received: 17 April 2012 / Accepted: 16 August 2012 /Published online: 4 September 2012

(C) Springer-Verlag 2012

\begin{abstract}
The Rho GTP exchange factor, Pebble (Pbl), long recognised as an essential activator of Rho during cytokinesis, also regulates mesoderm migration at gastrulation. Like other cell cycle components, $p b l$ expression patterns broadly correlate with proliferative tissue. Surprisingly, in spite of its role in the early mesoderm, $p b l$ is downregulated in the presumptive mesoderm before ventral furrow formation. Here, we show that this mesoderm-specific repression of $p b l$ is dependent on the transcriptional repressor Snail (Sna). $p b l$ repression was lost in sna mutants but was unaffected when Sna was ectopically expressed, showing that Sna is necessary, but not sufficient, for $p b l$ repression. Using DamID, the first intron of $p b l$ was identified as a Snabinding region. Nine sites with the Sna-binding consensus motif CAGGT[GA] were identified in this intron. Mutating these to TAGGC[GA] abolished the ventral repression of $p b l$. Surprisingly, Sna-dependent repression of $p b l$ was not essential for viability or fertility. Loss of repression did, however, increase the frequency of low-penetrance gastrulation defects. Consistent with this, expression of a $p b l-G F P$
\end{abstract}

Communicated by Claude Desplan

Electronic supplementary material The online version of this article (doi:10.1007/s00427-012-0414-8) contains supplementary material, which is available to authorized users.

M. J. Murray $\cdot$ W. Liu $\cdot$ H. Fraval $\cdot$ N. Lorensuhewa $\cdot$ R. Saint Research School of Biology, Australian National University, ACT 2601, Canberra, Australia

M. J. Murray $(\bowtie) \cdot$ H. Fraval $\cdot$ N. Lorensuhewa $\cdot$ R. Saint Genetics Department, University of Melbourne,

Victoria 3010, Melbourne, Australia

e-mail: murraym@unimelb.edu.au

T. D. Southall $\cdot$ A. H. Brand

Gurdon Institute and Department of Physiology, Development and Neuroscience, University of Cambridge,

Cambridge, UK transgene in the presumptive mesoderm generated similar gastrulation defects. Finally, we show that a cluster of Snailbinding sites in the middle of the first intron of $p b l$ orthologues is a conserved feature in the other 11 sequenced Drosophila species. We conclude that $p b l$ levels are precisely regulated to ensure that there is enough protein available for its role in early mesoderm development but not so much as to inhibit the orderly progression of gastrulation.

Keywords Snail $\cdot$ Pebble $\cdot$ Gastrulation $\cdot$ Rho GTP exchange factor

\section{Introduction}

During embryonic development, genes can act at multiple times and locations and can perform different molecular and cellular functions in different contexts. The complex functional requirements for a gene can be reflected by intricate and dynamic patterns of expression, which are under tight genetic regulation. The need for precise spatiotemporal control of gene expression is particularly acute in the developing embryo of the fly, where gene regulatory events must be precisely controlled over short time frames as the developmental events unfold in rapid succession. In this paper, we investigate one aspect of the regulation of a gene with pleiotropic functions: the Rho GTP exchange factor, Pebble ( $\mathrm{Pbl})$.

$\mathrm{Pbl}$ has long been known as an essential activator of the Rho signalling pathway that controls the formation and constriction of the cytokinetic actomyosin contractile ring (Prokopenko et al. 1999). Consistent with its role in cell cycle progression, $p b l$ is upregulated in tissues that are proliferating or have proliferative potential (Prokopenko et al. 2000). Given its role in such a fundamental aspect of cell biology, it was surprising, therefore, when it was discovered that $\mathrm{Pbl}$ is also required for mesoderm migration (Smallhorn 
et al. 2004; Schumacher et al. 2004). The Drosophila mesoderm is formed by the invagination of a ventral band of epithelial cells during gastrulation. Following internalisation, the epithelial tube flattens down onto the basal surface of the ectoderm, cells become more rounded and dissociated as the epithelial tube undergoes an epithelial to mesenchymal transition (EMT) and, finally, cells extend cellular protrusions as they migrate out over the ectoderm. Each of these processes is dependent on $\mathrm{Pbl}$ function (Smallhorn et al. 2004).

One puzzling aspect of the expression pattern of $p b l$ has been that, in spite of its role in early mesodermal development, it is actually repressed in the presumptive mesodermal cells prior to furrowing (Smallhorn et al. 2004). Gastrulation is extremely rapid in Drosophila: the progression from initial furrowing to the onset of migration occurs in less than an hour. It is counter-intuitive, therefore, that a critical mediator of mesodermal behaviour should be repressed just before it is required. In this study, we investigate $p b l$ repression in the presumptive mesoderm.

When considering how $p b l$ might be repressed, an obvious candidate is the transcription factor Snail (Sna). Snail family transcription factors are well known for their roles in promoting mesenchymal cell fates through repression of epithelial genes such as DECadherin. In Drosophila, Sna is specifically upregulated in the presumptive mesoderm by the master mesodermal factor Twist and is required for repression of a number of genes, including the DECad orthologue, shotgun (Leptin 1999). In addition to inducing EMTs, Snail family members have also been linked with cell cycle control. In flies, Sna binds cell cycle regulators string and $c y c E$ (Southall and Brand 2009) and can repress string expression in neuroblasts (Ashraf and Ip 2001). Similarly, in vertebrates, Snail can block cell cycle progression by repressing cyclin D2 (Vega et al. 2004) and when EMT is induced in human breast cancer cells by expression of SNA1, genes associated with the cell cycle, including the $\mathrm{Pbl}$ orthologue, Ect2, are generally downregulated as the EMT proceeds (Vetter et al. 2009).

In this paper, we show that Sna directly represses $p b l$ and identify Sna-binding sites in the first intron of $p b l$ that are required for this repression. We subsequently show that while this repression is not essential for viability, it is required for optimal fidelity of gastrulation. The transcriptional regulation of $p b l$ expression in gastrulating embryos, therefore, appears

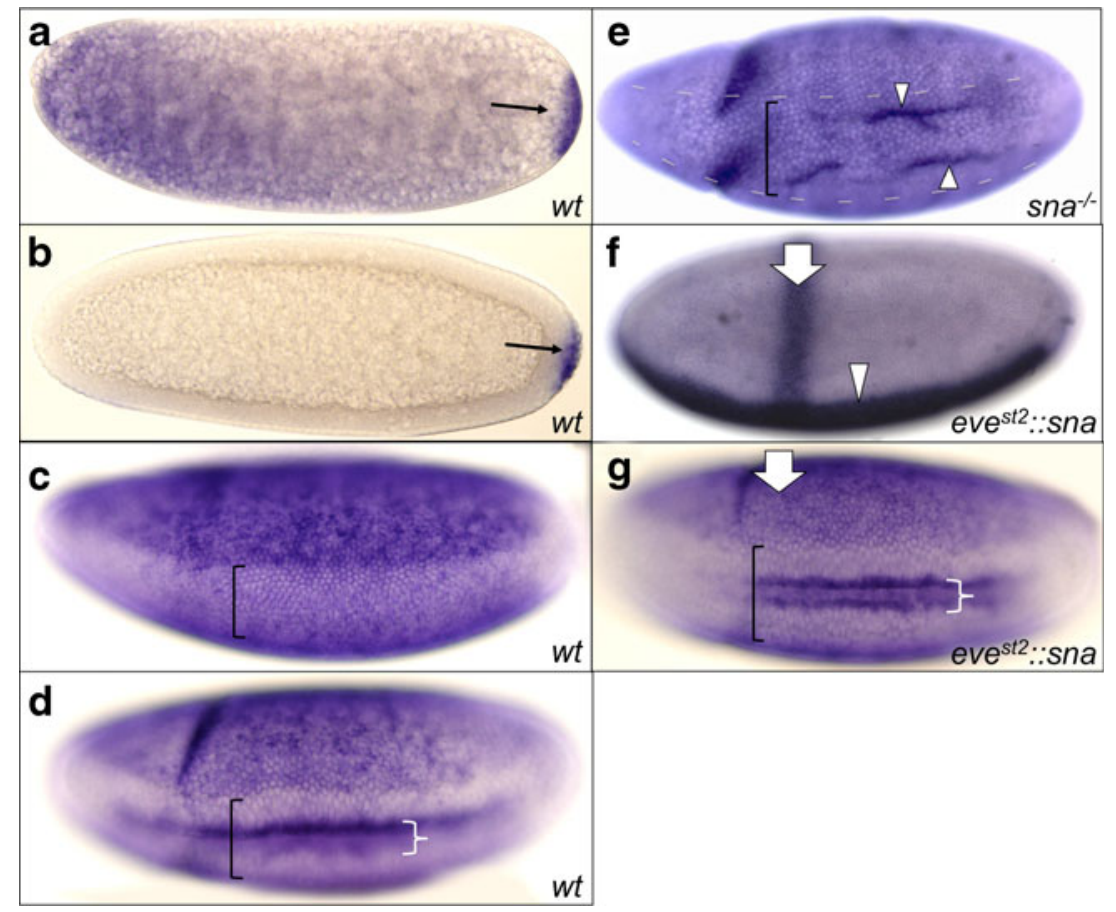

Fig. 1 Sna is necessary but not sufficient for repression of pbl. Wildtype $(\mathbf{a}-\mathbf{d}), s^{18} a^{18}(\mathbf{e})$ and $e v e^{s t 2}:$ sna $(\mathbf{f}, \mathbf{g})$ embryos stained for $p b l(\mathbf{a}-\mathbf{e}$, g) and sna mRNA (f). a-d. $p b l$ mRNA expression in early wild-type embryos. In preblastoderm embryos, $p b l$ is widely expressed but enriched at the posterior pole (a, arrow). By early stage 5, expression is confined to the pole cells (b, arrow). During stage 5, pbl is turned on throughout the ectoderm, but expression is significantly lower in a ventral band (c-d, black square brackets). Shortly before furrow formation and continuing as furrowing progresses, $p b l$ is de-repressed in a central region near the midline (d, curly brackets). A sna ${ }^{18}$ mutant embryo at a similar stage to $\mathbf{d}$ (e). The main ventral furrow has failed to form, but ectopic "mini-furrows" (arrowheads) are apparent. There is no repression of $p b l$ in the normal ventral furrow domain (region between dotted lines, black square bracket). An eve ${ }^{\text {st } 2}:$ sna embryo stained for sna mRNA, showing the normal band of ventral expression (arrowhead) and an ectopic band of expression corresponding to the eve stripe 2 region (arrow) (f). An eve $e^{s t 2}:$ sna embryo showing normal patterns of $p b l$ expression and no obvious ectopic band of $p b l$ repression in the eve stripe 2 domain (arrow) (g) 
to be a finely tuned compromise between a requirement for $\mathrm{Pbl}$ in mesodermal development on the one hand and impairment of gastrulation due to lack of repression on the other.

\section{Materials and methods}

\section{Drosophila genetics}

sna ${ }^{18}$ and $\beta 2$-tubulin-FLP fly stocks were obtained from the Bloomington stock centre. The eve-stripe2-FRT-STOPFRT-sna P-element construct (a gift from S. Small) (Nibu et al. 1998) was injected, and transformants are made by standard methods.

$p b l$ intron mutagenesis

To create att $\left[p b l^{W T}\right]$, att $\left[p b l^{\Delta S N A}\right]$, att $[p b l-G F P]$, and att $\left[p b l^{\Delta S N A}-G F P\right]$ transgenics, briefly, a genomic fragment containing $p b l$ and the two flanking genes was rescued by gap repair from the cosmid $\cos 34$ (G. Hime, unpublished) into $\mathrm{P}[\mathrm{acman}]$ to create $\mathrm{P}[\mathrm{acman}-p b l]$. The GFP sequence was then incorporated just prior to the STOP codon into P[acman- $p b l]$ using galK recombineering to create $\mathrm{P}[$ acman-pbl-GFP]. To mutate CAGGT[AG] Sna sites to TAGGC[AG], multiple mutagenesis primers were used in a multiple-mutation reaction, with Ampligase ${ }^{\circledR}$ Thermostable DNA Ligase (EPICENTRE Biotechnologies) and $P f u$ (Stratagene) at an annealing temperature of $45{ }^{\circ} \mathrm{C}$. The initial reaction successfully incorporated mutations to six of the motifs. We then used QuikChange ${ }^{\circledR}$ Site-directed Mutagenesis Kit (Stratagene) to introduce the mutations to the other three sites. Further details on primers and methodology are available on request. An unexpected A to $\mathrm{G}$ mutation was found in the final fragment, but this was located well away from the sna motifs. The mutated sequence was then incorporated into $\mathrm{P}[\mathrm{acman}-p b l]$ and $\mathrm{P}[\mathrm{acman}-p b l$ $G F P]$ using galK recombineering to create $\mathrm{P}\left[\right.$ acman- $p b l^{\Lambda S N A}$ ] and $\mathrm{P}\left[\right.$ acman- $\left.p b l^{\Delta S N A}-G F P\right]$. Both constructs were then injected into embryos expressing $\Phi \mathrm{C} 31$ to obtain insertions at the landing site $\mathrm{M}\{3 \times \mathrm{xP} 3-\mathrm{RFP}$.attP $\} \mathrm{ZH}-86 \mathrm{Fb}$ which were subsequently recombined onto $p b l^{2}$ and $p b l^{3}$ chromosomes.

In situ hybridisation, immunostaining and imaging

Immunohistochemistry and in situ hybridisation were performed using standard methods. Rabbit anti-Twist was used at 1:500, and Goat-anti-Rabbit-Alkaline Phosphatase at 1:500 (Jackson ImmunoResearch, West Grove, PA), Mouse-anti- $\beta$ galactosidase MAbs 40-1a at 1:100 (Developmental Studies Hybridoma Bank). Confocal images of twist::pblGFP embryos were captured on a Leica SP2 microscope. Image processing and 3-D rendering utilised ImageJ. In situ images were captured on an Olympus BX60 microscope and DP71 camera.
Embryonic lethality estimates

Lethality estimates of embryos were based on hatching rates of embryos laid from crosses attP( $p b l), p b l^{3} / T M 3 f t z l a c Z \times$ $a t t P(p b l), p b l^{2} / T M 3 f t z l a c Z$ and $a t t P\left(p b l^{\Delta S N A}\right), p b l^{3} /$ TM3ftzlacZ $\times \operatorname{attP}\left(p b l^{\Lambda S N A}\right), p b l^{2} / T M 3 f t z l a c Z$. Lethality was calculated as proportion of unhatched, fertilised embryos minus $25 \%$ (i.e. we assumed normal ratios of the four possible embryonic genotypes and full lethality of TM3ftzlacZ/ TM3ftzlacZ homozygotes). $\mathrm{N}$ values given in text are estimates of the number of embryos of the given genotype (i.e. $25 \%$ of total number).

\section{Results and discussion}

Sna is necessary but not sufficient for $p b l$ repression

To test whether Sna regulates $p b l$ expression, we compared $p b l$ mRNA in wild type and sna $a^{18}$ mutant embryos. In wild-type embryos, $p b l$ is initially expressed throughout the preblastoderm embryo but becomes enriched at the posterior end of the embryo (Fig. 1a) and confined to pole cells by stage 5 (Fig. 1b). During stage $5, p b l$ is turned on throughout the ectoderm, but its

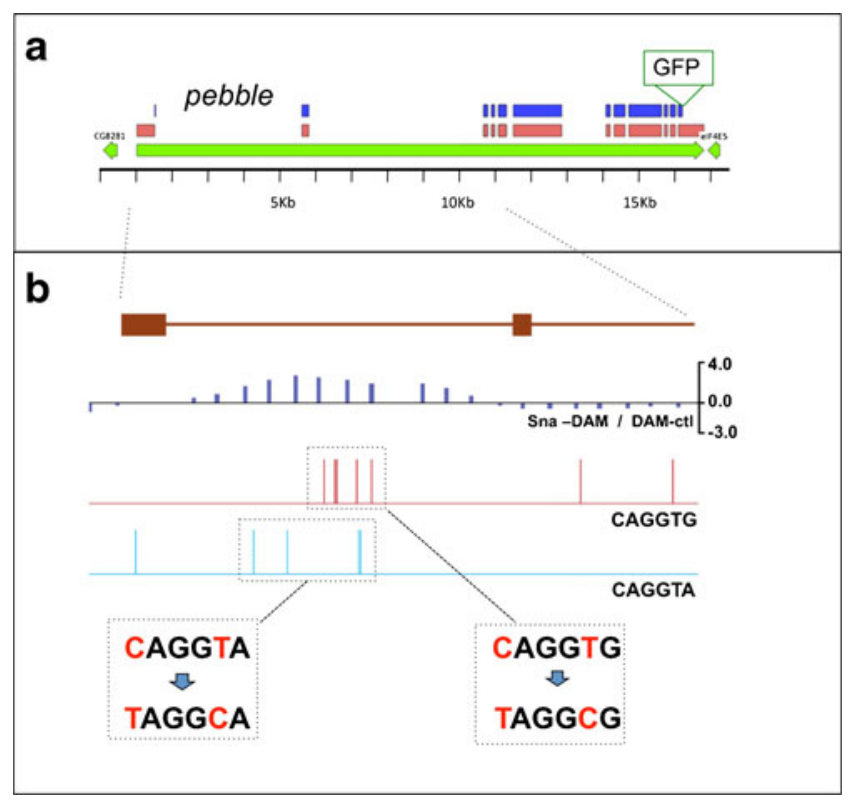

Fig. 2 Identification of Sna-binding sites at the $p b l$ locus. a Diagrammatic representation of the $p b l$ genomic region that was cloned into $\mathrm{p}$ [acman] and modified by the insertion of a C-terminal GFP tag. Green represents the $p b l$ transcript; pink, the exon structure; and blue, the open reading frame. b Binding of Dam-Sna in the first intron of $p b l$. Bar heights are proportional to the average of two normalised log2transformed ratio of intensities from DamID in vivo binding site experiments. The intron contains five CAGGTG (red) and four CAGGTA (blue) Sna consensus binding motifs. In the $p b l^{\Delta S N A}$ constructs, the CAGGTG and CAGGTA sites are mutated to TAGGCA and TAGGCG, respectively 


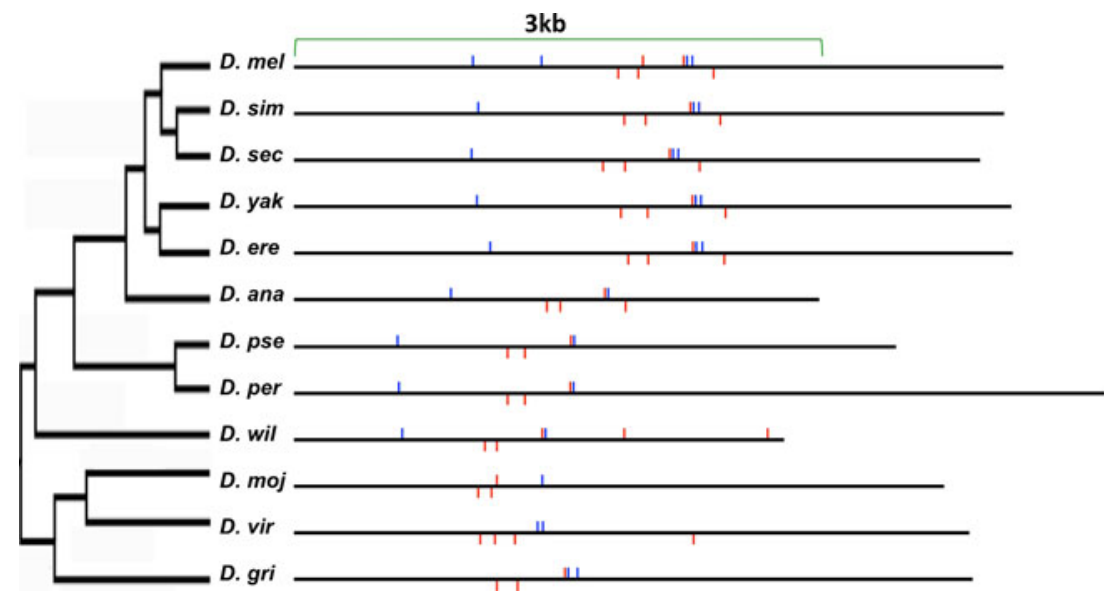

Fig. 3 Evolutionary conservation of Sna-binding sites in the first intron of $p b l$ in other Drosophila species. The positions of the Sna motif CAGGT[A/G] in the first introns of 11 Drosophila species $(D$. melanogaster (D. mel), D. simulans (D. sim), D. sechellia (D. sec), D. yakuba (D. yak), D. erecta (D. ere), D. ananassae (D. ana), D. pseudoobscura (D. pse), D. persimilis (D. per), D. willistoni (D. wil),

expression is partly repressed in a ventral band of the embryo (Fig. 1c, d; Smallhorn et al. 2004). pbl is also de-repressed in a narrow band near the midline just prior to, and during, furrow formation (Fig. 1d; Smallhorn et al. 2004).

In sna ${ }^{18}$ mutants, $p b l$ repression was abolished, with expression remaining constant throughout the ectoderm (Fig. 1e). Thus, Sna is necessary for $p b l$ repression.

To test whether Sna was sufficient for repression, we ectopically expressed Sna in the lateral ectoderm at a time
D. mojavensis (D. moj), D. virilis (D. vir) and D. grimshawi (D. gri)) are shown. Seven of the nine motifs in D. melanogaster are conserved in the closely related species, while a core cluster or four to five sites is conserved in even more distantly related species such as D. mojavensis and D. grimshawi. Red CAGGTG, blue CAGGTA. Ticks above and below the line indicate forward and reverse strands, respectively

when Sna is usually active in the presumptive mesoderm. We used the male specific $\beta 2$-tubulin-FLP to flipout the intervening STOP from the eve $e^{S T R I P E 2}>S T O P>$ sna construct and thereby induce sna expression in the even-skipped stripe 2 domain of stage 5 embryos.

As expected, the construct generated an ectopic stripe of sna expression (Fig. 1f), but $p b l$ expression was unaffected (Fig. $1 \mathrm{~g}$ ), even when the copy number of the eve STRIPE2 $>$ STOP $>$ sna construct was increased to four. Thus, Sna is

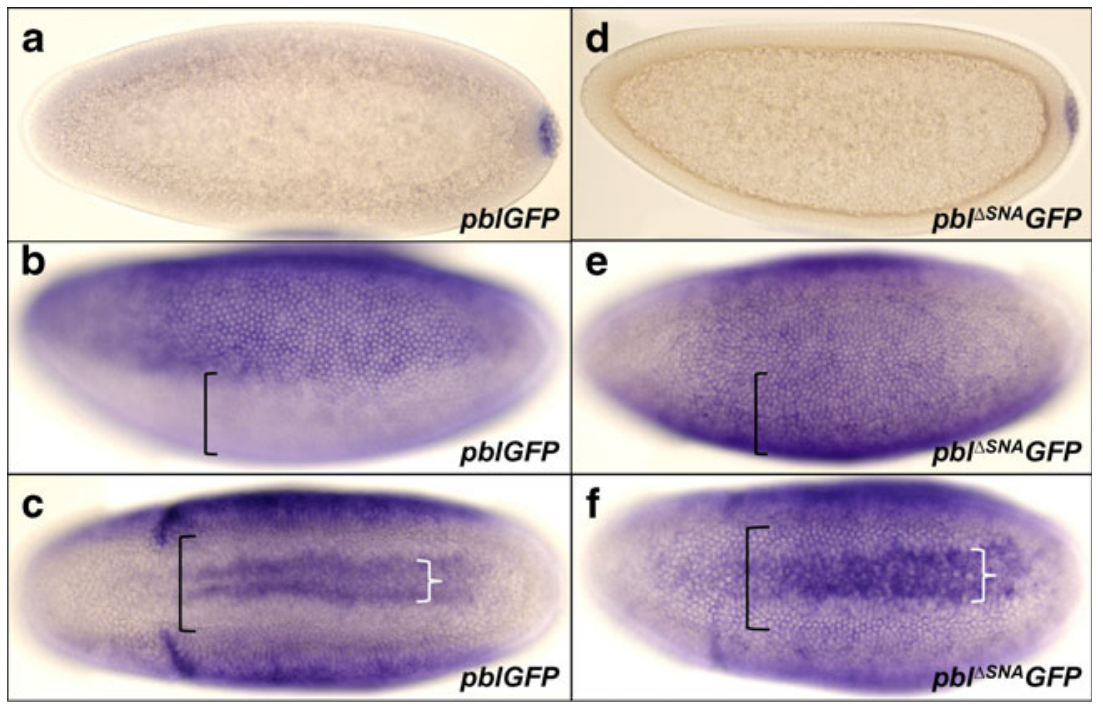

Fig. 4 Sna-binding sites are required for $p b l$ repression. a In situ stains for $G F P$ in embryos expressing $p b l-G F P(\mathbf{a}-\mathbf{c})$ and $p b l^{\Delta N A}-G F P(\mathbf{d}-\mathbf{f})$. a-c Embryos carrying the wild-type attP $[p b l-G F P]$ construct showing the normal pattern of $p b l$ expression at early stage 5 in pole cells (a), throughout the ectoderm but with a ventral band of repression (brackets) in stage 5 (b) and stage 6 (c) embryos and upregulation nearer the midline (c, white braces). d-f Embryos carrying the same construct in which the nine Sna consensus binding motifs have been mutated. As in control embryos, expression is seen in pole cells $(\mathbf{d})$ and throughout the ectoderm in stages 5 (e) and 6 (f) embryos, but there is no repression in the ventral domain (e, $\mathbf{f}$, black square brackets), though upregulation of $\mathrm{pbl}$ near the midline still occurs (f, white braces) 

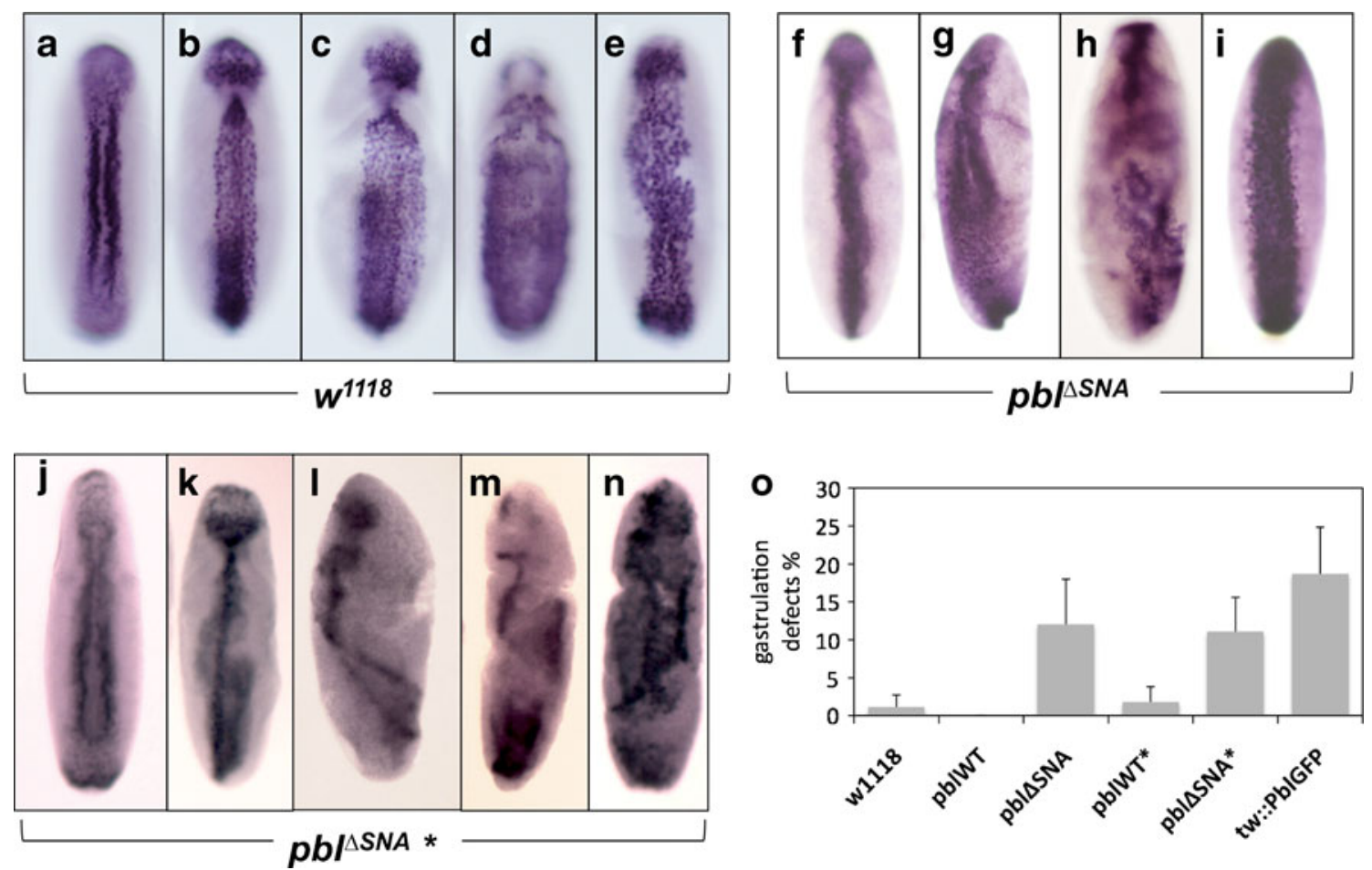

Fig. 5 Gastrulation defects in $p b l^{\Delta S N A}$ mutants. Embryos were immunostained for Twist to reveal mesoderm development. a-e $w 1118$ control embryos showing normal mesodermal invagination (a), dissociation (b) and spreading out into a monolayer (c-d). e An example of a small percentage ( $2 \%$ ) of $w 1118$ embryos in which the invaginated mesoderm shows unevenness. f-n $p b l^{\Delta S N A}$ embryos derived from parents with one $p b l$ mutant chromosome $(\mathbf{f}-\mathbf{i})$ and $p b l^{\Delta S N A *}$ embryos

necessary, but not sufficient, for repression of $p b l$. This was unexpected since the putative Sna co-repressor dCtBP is maternally expressed and would therefore be expected to be present in the lateral ectoderm and suggests that there exists some other factor necessary for repression, which may not be present outside the mesoderm region.

\section{Identification of Sna-binding sites in the first intron of $p b l$}

In a genome-wide DamID analysis of Sna-binding sites (Southall and Brand 2009), the first intron of $p b l$ was identified as a Sna-binding region (Fig. 2b). In vitro analyses of Sna-binding consensus sequences, and ChIP-chip de novo motif discovery, have previously identified CAGGTG as a highly conserved core binding motif (Mauhin et al. 1993); (Zeitlinger et al. 2007). Consistent with this, five CAGGTG sites were identified in close proximity to the DamID peak (Fig. 2b).

To identify further binding motifs, we have previously developed the MICRA software tool (Southall and Brand 2009) which identifies sequences that are overrepresented in genomic regions with DamID peaks. This analysis revealed that although the CAGGTG binding motif was indeed derived from $p b l^{\Delta S N A}$ parents $(\mathrm{j}-\mathrm{n})$, showing examples of the approximately $10 \%$ of embryos exhibiting gastrulation defects. These included an irregular/wavy internalised mesoderm (f, k), variations in the progression of gastrulation along the anterior-posterior axis $(\mathbf{g}, \mathbf{h}, \mathbf{j}, \mathbf{l})$, twisting $(\mathbf{g}, \mathbf{h}, \mathbf{l}, \mathbf{m})$, and severe failures of internalisation (i, $\mathbf{n})$. $\mathbf{o}$ Chart showing total proportions of gastrulation defects for different genotypes. Error bars show $95 \%$ confidence interval

enriched (43\% relative to background levels) in Dam-Snabinding regions, the enrichment of the motif CAGGTA was higher (71\% relative to background levels). Interestingly, the CAGGTA sequence was also found to efficiently bind Sna in vitro (Mauhin et al. 1993). Four CAGGTA motifs were located near the DamID peak (Fig. 2b).

Thus, Sna binds to the first intron of $p b l$, and this intron has nine potential Sna-binding sites.

\section{Evolutionary conservation of Sna-binding sites}

We next tested whether the enrichment of Sna motifs was conserved in related species. We examined the location of CAGGT[GA] motifs in the first intron of $p b l$ orthologues in 11 other species of Drosophila. In each case, the arrangement, size and spacing of exons of the $p b l$ locus was preserved, allowing us to unambiguously identify the first intron. In all Drosophila species, a cluster of four to five motifs towards the centre of the intron was conserved, including the more distantly related species such as Drosophila psuedoobscura and Drosophila grimshawi (Fig. 3). Analysis of five other arbitrarily chosen motifs showed conservation out to Drosophila erecta but not for the more distantly related species (Fig. S1). 
Thus, the cluster of Sna-binding sites in $p b l$ intron 1 has been conserved throughout the Drosophila genus.

Next, we checked whether Sna repression of $p b l$ was conserved in a much more distantly related insect, the mosquito. Although this might seem unlikely, genetic regulation of dorsoventral patterning in Anopheles gambiae shows surprising levels of conservation with flies, with key genes such as sna, having comparable patterns of expression to Drosophila (Goltsev et al. 2007). The $p b l$ orthologues in the mosquitos $A$. gambiae (AGAP005796) and Aedes aegypti (AAEL000984) had a similar genomic structure to Drosophila, though the first annotated exon in both cases clearly matched exon 2 of Drosophila (Fig. S2). Analysis of CAGGT[GA] motifs over the entire loci of AGAP005796 and AAEL000984 (including $20 \mathrm{~kb}$ upstream sequence) did not reveal a cluster of Sna motifs similar to that found in Drosophila. Interestingly, in the mosquito, internalisation of presumptive mesodermal cells does not proceed by a whole-scale furrowing event but, rather, by the ingression of individual mesodermal cells (Goltsev et al. 2007). The lack of Sna motifs, therefore, may be indicative of a different mode of gastrulation.

The Sna-binding sites in the first intron of $p b l$ are required for Sna-mediated repression

To test whether these nine sites were functionally important, we generated genomic rescue constructs in which they were mutated. We cloned a 17.5-kb genomic region encompassing the $p b l$ gene and the two flanking genes (Fig. 2a). This construct, $\mathrm{P}[\mathrm{acman}-p b l]$, was inserted onto the third chromosome and introduced into a $p b l^{3} / p b l^{2}$ null mutant background. The construct was able to completely rescue the known cytokinetic and mesoderm migration phenotypes of $p b l$ mutants (data not shown), and attP $\left[p b l^{W T}\right], p b l^{2} /$ attP $\left[p b l^{W T}\right]$, and $p b l^{3}$ (hereafter $p b l^{W T}$ ) adults were viable and fertile.

We mutated the nine sites to see if they were required for the normal ventral repression of $p b l$ expression. To achieve strong disruption to Sna binding with minimum DNA changes, we mutated just two highly conserved bases within each binding site. Based on published Sna-binding motifs (Ip et al. 1992; Mauhin et al. 1993; Zinzen et al. 2006) and in vitro mutation tests in which individual bases were mutated (Mauhin et al. 1993), we chose the $C$ in position 1 and the $T$ in position 5 as the bases to be mutated to $\mathrm{T}$ and $\mathrm{C}$, respectively, to create the construct $\mathrm{P}\left[\right.$ acMan- $\left.p b l^{\Delta S N A}\right]$ (Fig. 2b).

To examine the transcriptional expression pattern of this construct, without the complication of endogenous $\mathrm{pbl}$ mRNA, we tagged both $p b l^{W T}$ and $p b l^{\Delta S N A}$ with GFP to create $\mathrm{P}\left[\right.$ acman- $\left.p b l^{W T} G F P\right]$ and $\mathrm{P}\left[\right.$ acman- $\left.p b l^{\Delta S N A}-G F P\right]$. These constructs were introduced into the same attP site on the third chromosome as that used for the wild-type constructs, and expression of the GFP fusion transgenes was assessed with in situ hybridisation to GFP. $p b l^{W T} G F P$ was expressed in the same pattern as endogenous $p b l$ with pole-cell expression (Fig. 4 a), general ectodermal expression with downregulation in a ventral band (Fig. 4b, c) and upregulation near the midline (Fig. 4c). In contrast, $p b l^{\Delta S N A}-G F P$ was expressed throughout the ectoderm with no ventral repression either before (Fig. 4e) or during gastrulation (Fig. 4f). Other endogenous patterns of $p b l$ such as the expression in pole cells (Fig. 4d), upregulation near the midline (Fig. 4f) and in the longitudinal visceral muscle precursors (data not shown) were normal, indicating that the mutations did not wholly disrupt $p b l$ regulation. Thus, one or more of the nine Sna consensus sites are required for repression of $p b l$ in the presumptive mesoderm.

Loss of Sna repression of $p b l$ increases the frequency of gastrulation defects

Next, we tested the functional consequences of loss of $p b l$ repression. The $p b l^{\Delta S N A}$ rescue construct described above was recombined with $p b l^{2}$ and $p b l^{3}$ mutant alleles. Surprisingly attP $\left[p b l^{\Delta S N A}\right], p b l^{2} / \operatorname{attP}\left[p b l^{\Delta S N A}\right], p b l^{3}$ (hereafter $p b l^{\Delta S N A}$ ) flies were also viable and fertile, showing that Sna repression of $p b l$ is not essential.

To test whether loss of Sna repression of $p b l$ might nevertheless affect gastrulation, we immunostained embryos for the mesodermal marker Twist. In $w^{1118}$ control embryos, the great majority of embryos $(98.9 \%, n=174)$ had normal gastrulation (Fig. 5a-d), though two embryos showed an irregular invaginated tube (Fig. 5e). $p b l^{\Lambda S N A}$ embryos were more frequently disrupted with defects in $12.1 \%(n=116)$ of embryos (Fig. $5 f-$ i). In contrast, $p b l^{W T}$ embryos were all normal $(n=112)$. Gastrulation defects seen in $p b l^{\Delta S N A}$ embryos were (a) an irregular "wavy" invaginated mesodermal tube ( $n=5$; Fig. 5f), (b) regions of delayed invagination along the anterior-posterior axis and twisting ( $n=4$; Fig. $5 \mathrm{~g}, \mathrm{~h})$, and (c), a severe failure to internalise mesodermal cells ( $n=5$; Fig. 5i). Embryonic lethality was also increased from $\sim 5 \%\left(n=\sim 192^{1}\right)$ in $p b l^{W T}$ embryos to $\sim 9 \%(n=\sim 268)$ in $p b l^{\Delta S N A}$.

Since $p b l^{\triangle S N A}$ and $p b l^{W T}$ flies were viable and fertile, we were also able to examine embryos derived from $p b l^{W T}$ or $p b l^{\Delta S N A}$ parents (hereafter $p b l^{W T^{*}}$ and $p b l^{\Delta S N A^{*}}$ embryos). Again, gastrulation defects were more common in $p b l^{\Lambda S N A^{*}}$ $(11.1 \% n=189)$ embryos than $p b l^{W T^{*}}(1.8 \% n=166) \mathrm{em}-$ bryos. These frequencies were not significantly different than those from embryos derived from heterozygous parents with one wild-type copy of $p b l$ (two-tailed Fisher's exact test), and the range of defects was comparable (Fig. $5 j-n$ ). This result implies that gastrulation defects are associated only with zygotic, not maternal, loss of $p b l$ Sna motifs.

\footnotetext{
${ }^{1}$ See Materials and Methods.
} 

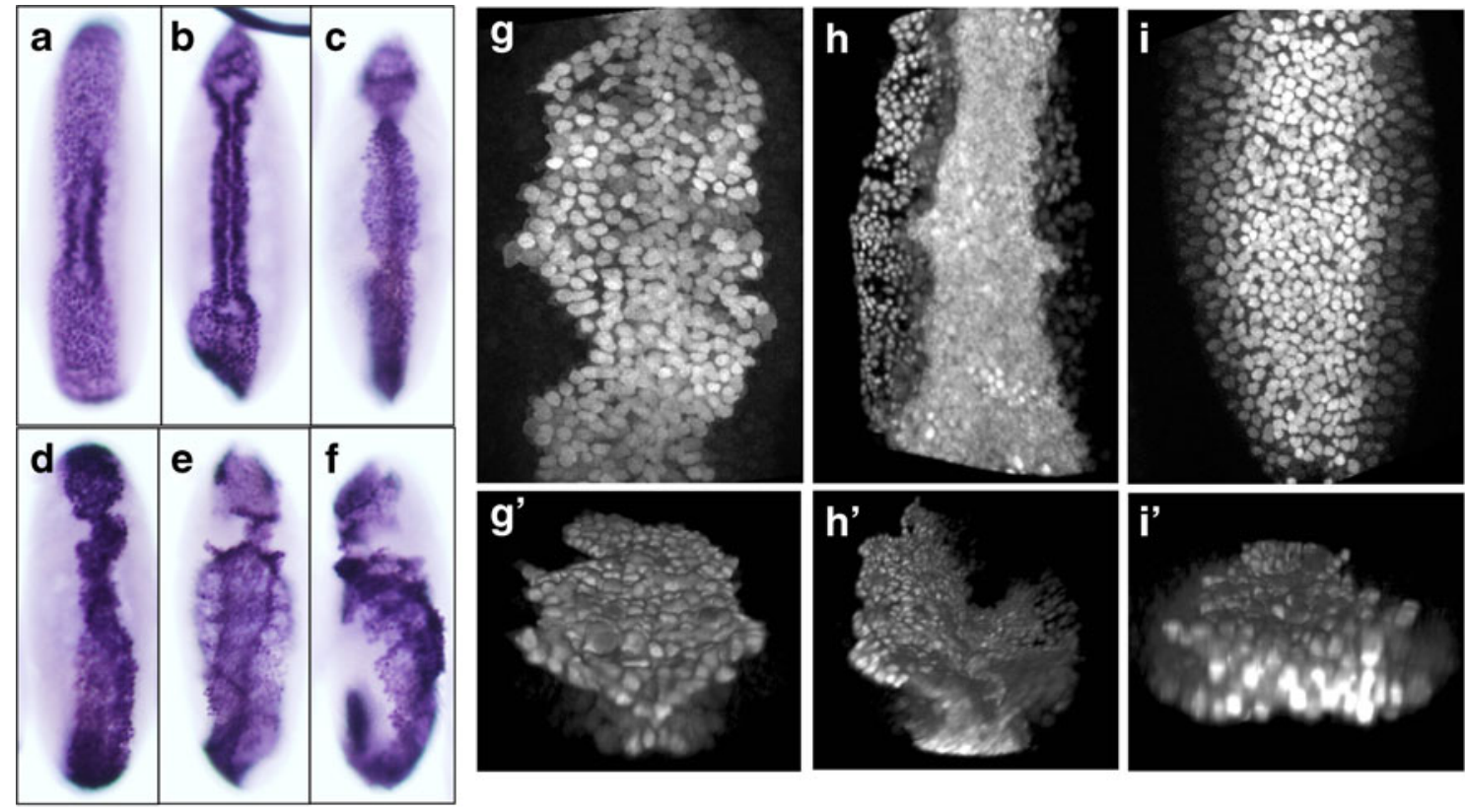

\section{twist::PbIGFP}

Fig. 6 Gastrulation defects in twist::pblGFP embryos. Embryos expressing twist::PblGFP stained for Twist (a-f) or GFP (g-i) showing gastrulation defects, which are similar to those observed in $p b l^{\Delta S N A}$ embryos (Fig. 5) though more penetrant. These included variations in progression of gastrulation along the anterior-posterior axis $(\mathbf{a}, \mathbf{b}, \mathbf{d})$, twisting $(\mathbf{b}, \mathbf{f})$, irregular/wavy mesoderm $(\mathbf{c}, \mathbf{g})$ and severe internalisation defects $(\mathbf{e}, \mathbf{h}, \mathbf{i}) . g^{\prime}, h^{\prime}$ and $i^{\prime}$ show three-dimensional renderings of $\mathbf{g}, \mathbf{h}$ and $\mathbf{i}$, viewed from the anterior end of the embryo
Finally, we directly tested whether increased levels of $\mathrm{Pbl}$ in the presumptive mesoderm could affect gastrulation. To obtain transgene expression prior to furrowing, we fused the twist promoter to a $p b l$ transgene tagged with a C-terminal GFP. We know that PblGFP is a functional transgene since it is able to rescue both cytokinesis and mesoderm migration defects of $p b l$ mutants (data not shown), and the PblGFP genomic rescue construct described earlier can rescue $p b l$ null mutants to viability.

In twist::pblGFP embryos PblGFP was expressed in a pattern that recapitulated endogenous twist patterns (e.g.
Fig. $6 \mathrm{~g}$ and data not shown), and homozygous twist:: pblGFP flies (in a wild-type background) were viable and fertile. twist::PblGFP embryos exhibited similar gastrulation defects to $p b l^{\triangle S N A}$ embryos though they were more penetrant (18.7\%, $n=155)$ (Fig. 6 and Table 1). We conclude that loss of Sna-mediated $p b l$ repression significantly increases the frequency of gastrulation defects, most likely due to increased levels of Pbl.

Although Pbl has not previously been linked with ventral furrowing, apical constriction of the invaginating mesoderm is a Rho-dependent process requiring correct apical

Table 1 Gastrulation defects in $p b l^{\Delta S N A}$ embryos

\begin{tabular}{|c|c|c|c|c|}
\hline Genotype & Number & Defects & Percent & $p$ value \\
\hline$w^{1118}$ & 174 & 2 & 1.1 & - \\
\hline$a t t P(P b l), p b l^{3} / a t t P(P b l), p b l^{2}$ & 112 & 0 & 0 & $0.5218^{\mathrm{a}}$ \\
\hline $\operatorname{att} P\left(P b l^{\Delta S N A}\right), p b l^{3} / a t t P\left(P b l^{\Delta S N A}\right), p b l^{2}$ & 116 & 14 & 12.1 & $<0.0001^{\mathrm{b}}$ \\
\hline $\operatorname{att} P(P b l), p b l^{3} / a t t P(P b l), p b l^{2} *$ & 166 & 3 & 1.8 & $0.2747^{\mathrm{b}}$ \\
\hline $\operatorname{att} P\left(P b l^{\Delta S N A}\right), p b l^{3} / a t t P\left(P b l^{\Delta S N A}\right), p b l^{2} *$ & 189 & 21 & 11.1 & $0.0011^{\mathrm{c}}$ \\
\hline twist::PblGFP & 155 & 29 & 18.7 & $<0.0001^{\mathrm{a}}$ \\
\hline
\end{tabular}

*The given genotype is that of the parents (see text)

${ }^{a} p$ value represents two-tailed Fisher's exact test with respect to $w 1118$

${ }^{\mathrm{b}} p$ value represents two-tailed Fisher's exact test with respect to $\operatorname{att} P(P b l), p b l^{3} / a t t P(P b l), p b l^{2}$

${ }^{\mathrm{c}} p$ value represents two-tailed Fisher's exact test with respect to att $P(P b l), p b l^{3} / a t t P(P b l), p b l^{2}$ * 
localisation of another RhoGEF, RhoGEF2 (Hacker and Perrimon 1998). When expressed in early mesodermal cells PblGFP localises evenly around the cell cortex. We speculate, therefore, that higher levels of $\mathrm{Pbl}$, might alter the balance of apical/basal Rho-dependent cortical contractile forces, which disrupts the apical contraction of cells leading to occasional failures in furrowing.

In conclusion, we have shown that Sna represses transcription of $p b l$ in the mesoderm primordium of $D$. melanogaster via one or more Sna-binding sites, which are conserved among species of the Drosophila genus, but not in the mosquito, correlating with the different modes of gastrulation in the different genuses. Although Snamediated repression of $p b l$ was found not to be essential for viability, the selective pressure to maintain the mechanism is likely to come from our observation that loss of repression of $p b l$ in the mesoderm primordium disrupts furrowing and lowers viability. Following $p b l$ repression in the mesoderm primordium, $p b l$ is again upregulated near the centre of the presumptive mesoderm just prior to furrowing. This upregulation may be required to reinstate the levels of $\mathrm{Pbl}$ required for mesoderm migration and the cytokineses that occur during the migration phase. The genetic basis for this upregulation awaits further study. These results speak to the importance of tight spatiotemporal control in fine tuning developmental events so as to maximise the efficiency of the developmental process.

Acknowledgments We thank Steve Small for the eve-stripe2-snail construct and helpful discussions on the Snail-binding sites mutation strategy; Maria Leptin, for the gift of anti-Twist antibody; Michael Akam, for the gift of the twist-GAL4 plasmid; and the Bloomington Stock Center and the Developmental Studies Hybridoma Bank for stocks and antibodies. This work was funded by an Australian Research Council Discovery Grant to RS and a Wellcome Trust Programme Grant (programme grant 068055) and MRC Project Grant to AHB. AHB acknowledges the core funding provided by the Wellcome Trust (092096) and CRUK (C6946/A14492). TDS was funded by a Herchel Smith Fellowship.

\section{References}

Ashraf SI, Ip YT (2001) The Snail protein family regulates neuroblast expression of inscuteable and string, genes involved in asymmetry and cell division in Drosophila. Development 128:4757-4767
Goltsev Y, Fuse N, Frasch M, Zinzen RP, Lanzaro G, Levine M (2007) Evolution of the dorsal-ventral patterning network in the mosquito, Anopheles gambiae. Development 134 (13):2415-2424

Hacker U, Perrimon N (1998) DRhoGEF2 encodes a member of the $\mathrm{Dbl}$ family of oncogenes and controls cell shape changes during gastrulation in Drosophila. Genes Dev 12(2):274-284

Ip YT, Park RE, Kosman D, Bier E, Levine M (1992) The dorsal gradient morphogen regulates stripes of rhomboid expression in the presumptive neuroectoderm of the Drosophila embryo. Genes Dev 6(9):1728-1739

Leptin M (1999) Gastrulation in Drosophila: the logic and the cellular mechanisms. EMBO J 18(12):3187-3192

Mauhin V, Lutz Y, Dennefeld C, Alberga A (1993) Definition of the DNA-binding site repertoire for the Drosophila transcription factor SNAIL. Nucleic Acids Res 21(17):3951-3957

Nibu Y, Zhang H, Bajor E, Barolo S, Small S, Levine M (1998) dCtBP mediates transcriptional repression by Knirps, Krüppel and Snail in the Drosophila embryo. EMBO J 17(23):70097020

Prokopenko SN, Brumby A, O'Keefe L, Prior L, He Y, Saint R, Bellen HJ (1999) A putative exchange factor for Rhol GTPase is required for initiation of cytokinesis in Drosophila. Genes Dev 13 (17):2301-2314

Prokopenko SN, Saint R, Bellen HJ (2000) Tissue distribution of PEBBLE RNA and Pebble protein during Drosophila embryonic development. Mech Dev 90:269-273

Schumacher S, Gryzik T, Tannebaum S, Müller H-AJ (2004) The RhoGEF Pebble is required for cell shape changes during cell migration triggered by the Drosophila FGF receptor Heartless. Development 131(11):2631-2640

Smallhorn M, Murray MJ, Saint R (2004) The epithelialmesenchymal transition of the Drosophila mesoderm requires the Rho GTP exchange factor Pebble. Development 131 (11):2641-2651

Southall TD, Brand AH (2009) Neural stem cell transcriptional networks highlight genes essential for nervous system development. The EMBO Journal:1-9.

Vega S, Morales AV, Ocaña OH, Valdés F, Fabregat I, Nieto MA (2004) Snail blocks the cell cycle and confers resistance to cell death. Genes Dev 18(10):1131-1143

Vetter G, Béchec AL, Muller J, Muller A, Moes M, Yatskou M, Tanoury ZA, Poch O, Vallar L, Friederich E (2009) Timeresolved analysis of transcriptional events during SNAI1triggered epithelial to mesenchymal transition. Biochem Biophys Res Commun 385(4):485-491

Zeitlinger J, Zinzen RP, Stark A, Kellis M, Zhang H, Young RA, Levine M (2007) Whole-genome ChIP-chip analysis of Dorsal, Twist, and Snail suggests integration of diverse patterning processes in the Drosophila embryo. Genes Dev 21 (4):385-390

Zinzen RP, Senger K, Levine M, Papatsenko D (2006) Computational models for neurogenic gene expression in the Drosophila embryo. Curr Biol 16(13):1358-1365 